\title{
The short-term outcome of severe blunt head injury as reported by relatives of the injured persons
}

\author{
W W M C I NLAY, D N B ROKS, M R B OND, \\ D P M A R I N A E, A N D M MAR HALL \\ From the Department of Psychological Medicine, University of Glasgow, Glasgow, Scotland
}

S UMMARY A close relative of 55 severely head injured adults (post-traumatic amnesia $\geqslant 2$ days) was interviewed 3, 6 and 12 months after injury to obtain information about psychosocial changes in the patient. The problems most frequently reported were emotional disturbances, poor memory, and subjective symptoms, with physical disability much less common. The amount of stress experienced by relatives did not diminish between 3 and 12 months, and was related to the incidence of mental and behavioural changes in the patient. The question of whether or not compensation was being claimed did not appear to influence the outcome.

Improvements in medical care have made it increasingly likely that patients with severe blunt head injuries will survive. However, a considerable number will remain handicapped and these place a burden on their families and a demand on rehabilitation services. ${ }^{1-4}$ While much research has appeared on cognitive deficits, especially memory deficits, ${ }^{7}$ there has been less emphasis on the broader spectrum of "psychosocial" changes which may follow head injury. These psychosocial sequelae of head injury include cognitive, emotional and behavioural changes and their effects on family, leisure and occupational life. Reports on the severely injured patient's physical and mental state, however, have stressed the importance of mental changes and in particular personality change. Fahy et $a l^{6}$ found "psychiatric symptoms" in 17 out of 22 cases who had post-traumatic amnesia (PTA) of more than three days. Thomsen $^{7}$ not only found personality change in 42 out of 50 cases but noted that patients often lack insight, and for this reason researchers have often interviewed relatives to obtain information about changes in the patient.

Relatives of the brain injured often experience stress, ${ }^{89}$ although parent-child relationships have been considered to be more resilient than husbandwife relationships. ${ }^{710}$ The mental changes in the patient following injury, especially personality

Address for reprint requests: Mr WW McKinlay, University of Glasgow, Department of Psychological Medicine, 6 Whit tingehame Gardens, Glasgow G12 0AA.

Accepted 28 December 1980 changes and memory deficits, tend to cause families more difficulty than physical disability. ${ }^{2} 711$ Relatives may deny disability initially and go through an adjustment process analogous to grieving; ${ }^{12}$ and relatives may take some time to realise the full extent of the changes in the patient. ${ }^{13}$

The studies mentioned above have been concerned largely with the presence of difficulties and disabilities in the patient and the effects of these on close relatives. Other studies have assessed outcome in functional terms (resumption of activities, especially work), although large-scale followups which report nearly all patients returning to work may include many cases with very minor injuries. ${ }^{14}$ Oddy et $a l^{15}$ report that almost half of their "severely" injured patients had resumed full-time work, and another $15 \%$ part-time work, only four months after injury and that all of these had resumed work at the same level as before injury. However, it must be noted that the sample of Oddy et al was young $(80 \%$ under 25 years of age), with more cases from the upper than lower social strata and that half the cases had PTA of seven days or less, all of which make a good outcome more likely. Many of the previous studies are unsatisfactory and Oddy $e a a^{15}$ noted that it was often difficult to draw clear conclusions owing to inadequate specification of selection criteria for patients, and inadequate description of study populations, especially with regard to severity. Accordingly, the present authors aim to provide data on the mental, physical and behavioural 
changes observed in a well-documented group of patients. The observers were close relatives of the injured persons and were interviewed at 3, 6 and 12 months after injury and asked to report changes which had occurred in the patient consequent on the injury. Two general questions are posed: (a) what changes in the patient are most frequently observed in the 12 months following injury? and (b) which particular changes are reported in cases where the caring relatives experience stress?

The data presented here are based on part of a continuing and wider study of the psychological and social consequences of severe blunt head injury, some reports of which have already been published. ${ }^{417}$

\section{Method}

\section{Patients}

Fifty-five cases (46 male) aged 16-60 years (mean $35 \cdot 7$, SD 14.3) with severe blunt head injury constituted the sample. "Severe" injury was defined by at least two days post-traumatic amnesia (PTA) and "blunt" injury was defined as follows: those with penetrating localised wounds were excluded but all other head injuries, including those with depressed fractures, were admitted to the study. Patients known to have previous neurological disease or damage were excluded. The present sample was drawn from the Institute of Neurological Sciences (INS) in Glasgow, which is a secondary facility serving the West of Scotland, and patients are transferred there for investigations and treatments not available at the referring hospitals. The fact that the sample is drawn from a secondary facility necessitates as full a

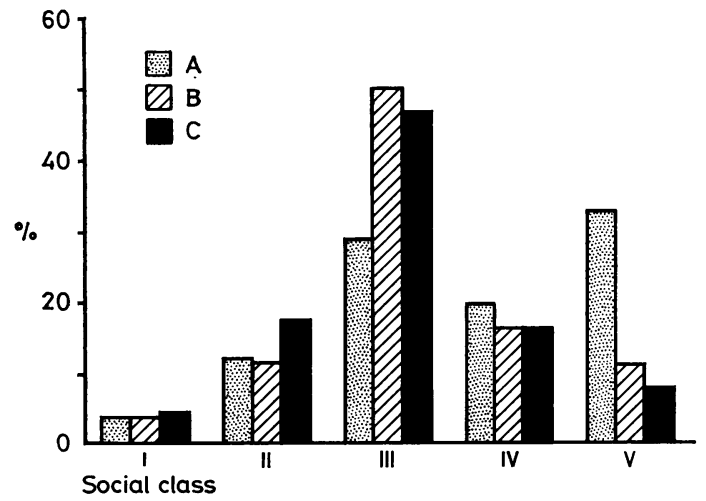

Fig 1 Social class distribution: percentage of patients $(A)$ in each of the Registrar General's social classes compared with the general population of the Clydeside conurbation $(B)$ and the United Kingdom as a whole $(C)$. description as possible of demographic and clinical indices to make clear the nature of the sample. Figure 1 and tables 1-4 provide such data. As defined by PTA the sample is a very severely injured one with only 12 out of 55 cases $(22 \%)$ having PTA of 2-7 days and the median value for PTA being 21 days. It will also be noted that the lower social classes are over-represented in comparison with the population of the catchment area of the present research, that is, the Clydesdale conurbation. This over-representation of the lower social classes is characteristic of the head-injured population. ${ }^{18}$ Nineteen patients were single, 33 were married or cohabiting, and one each was separated, divorced, and widowed.

Table 1 Location or cause of injury

\begin{tabular}{lr}
\hline Road traffic accident & 26 \\
Accident at work & 4 \\
Accident at home & 1 \\
Assault & 11 \\
"Fell" & 7 \\
Other & 6 \\
Total & 55 \\
\hline
\end{tabular}

Table 2 Major complications present

Skull fracture only

Haematoma only

Both skull fracture and haematoma

Neither

Total

13
11
18
13
55

Table 3 Whether neurosurgical operation was carried out

\begin{tabular}{ll} 
Yes & 28 \\
No & 27 \\
Total & 55 \\
\hline
\end{tabular}

Table 4 Duration of post traumatic amnesia (PTA)

\begin{tabular}{lc}
\hline $2-7$ days & 12 \\
$8-14$ days & 6 \\
$15-28$ days & 14 \\
$1-2$ months & 12 \\
$2-3$ months & 5 \\
Over 3 months & 4 \\
Total & $53^{*}$ \\
\hline
\end{tabular}

* In 2 cases no reliable estimate of PTA could be obtained.

Procedure

A close relative of each patient was interviewed three times. Interviews were carried out at 3,6 and 12 months post-injury. Relatives were asked to report changes in the patient which emerged after injury and which were present either since injury or since the previous assessment, whichever was the more recent. Interviewees who bore a major day-to-day responsibility for care of the patient (or would have if 
the patient was not in hospital), were included (see table 5). The structured interview schedule included questions about the patient's physical and mental state, behaviour and self-care ability. This was based on the methods used by Grad and Sainsbury ${ }^{19}$ and Hoenig and Hamilton ${ }^{20}$ : changes observed in the patient were designated "objective" burden and the amount of strain or distress experienced by the relative as arising from these "objective" changes was designated "subjective" burden.

Table 5 Relationship of informants to patients

\begin{tabular}{lccc}
\hline & 3 months & 6 months & 12 months \\
\hline Wife & 28 & 27 & 27 \\
Husband & 3 & 4 & 4 \\
Mother & 10 & 12 & 12 \\
Father & 5 & 6 & 5 \\
Other relative & 8 & 6 & 5 \\
Non relative & 1 & 0 & 2 \\
& 55 & 55 & 55 \\
\hline
\end{tabular}

Note: While every attempt was made to interview the same informant at each follow-up, this did not always prove possible. However, all informants bore a major day-to-day responsibility for care of the patient.

The interview schedule was designed to assess problems reported in the literature or encountered during the authors' clinical and research work with head injured patients. There were 90 items which made as comprehensive as possible a survey of the difficulties which may be observed in head injured patients, that is objective burden. Subjective burden was assessed using a simple seven-point rating scale ranging from: (1) "I feel no strain as a result of the changes in my spouse/relative" to (7) "I feel severe strain as a result of the changes in my spouse/ relative."

It is important to note that only changes (that is the appearance of mental or physical deficits or abnormal behaviours in the patient) occurring since injury were recorded. Moreover, ratings of both objective and subjective burden refer explicitly to the relative's view of the situation. Reliability of a pilot version of the questionnaire was assessed by having two researchers interview 20 relatives: 10 relatives were interviewed by one researcher and 10 by the other with both present. Disagreement occurred only where the degree rather than presence of change was recorded and questions where such disagreements occurred are not included in the present analysis.

\section{Results}

The most frequently reported changes in the patient were mental rather than physical with such difficulties as slowness, tiredness, irritability and poor memory being reported in the majority of cases (table 6). In order to obtain an overall view of the most prevalent difficulties, questionnaire items were divided into seven categories for
Table 6 The ten problems most frequently reported by relatives as being present in the patient (per cent reporting)

\begin{tabular}{llll}
\hline & 3 months & 6 months & 12 months \\
\hline Slowness & 86 & 69 & 67 \\
Tiredness & 82 & 69 & 69 \\
Irritability & 63 & 69 & 71 \\
Poor memory & 73 & 59 & 69 \\
Impatience & 60 & 64 & 71 \\
Tension and & 57 & 66 & 58 \\
anxiety & & 56 & \\
Bad temper & 48 & 58 & 67 \\
Personality change & 49 & 52 & 57 \\
Depressed mood & 57 & 46 & 53 \\
Headaches & 54 & & \\
\hline
\end{tabular}

analysis. These categories (listed below) are arbitrary to some degree, but an attempt was made to reflect common distinctions which are made in the literature and in clinical practice.

(1) The physical category included sensory and motor impairment, gait disturbances, fits and poor balance. The most common changes were impairment of vision (usually minor) quoted in $53 \%, 47 \%$ and $42 \%$ of cases at 3,6 and 12 months respectively; and an impairment of balance reported in $49 \%, 40 \%$ and $40 \%$ of cases. Paralysis of a limb was relatively uncommon $(11 \%, 6 \%$ and $4 \%$ at successive follow-ups), and even by 3 month follow-up most patients were independently mobile (see table 7).

Table 7 Degree of physical mobility

\begin{tabular}{|c|c|c|c|c|c|c|}
\hline \multirow{4}{*}{$\begin{array}{l}\text { Independently mobile } \\
\text { Independent, but needs } \\
\text { stick or crutch }\end{array}$} & \multicolumn{2}{|c|}{3 months } & \multicolumn{2}{|c|}{6 months } & \multicolumn{2}{|c|}{12 months } \\
\hline & $N$ & $\%$ & & $\%$ & $N$ & $\%$ \\
\hline & 48 & 87 & 50 & 91 & 50 & 91 \\
\hline & 5 & 9 & 3 & 6 & 3 & 6 \\
\hline Confined to wheelchair & 1 & 2 & 2 & 4 & 2 & 4 \\
\hline Confined to bed & 1 & 2 & 0 & 0 & 0 & 0 \\
\hline
\end{tabular}

(2) The language category consisted of dysarthria and dysphasia. The most frequent problems were difficulty in expression (for example word finding or fluency) reported in $47 \%$, $44 \%$ and $44 \%$ of cases at 3, 6 and 12 months respectively, and dysarthria in $33 \%, 26 \%$ and $29 \%$ of cases. In some cases the difficulties were minor and although noted by relatives were not always apparent to the psychologist who assessed the patient. Receptive difficulties were less common (no more than $15 \%$ of cases at any follow-up). There was an association between dysarthria and physical disability at 3 months, when five out of the six cases with 
paralysis of a limb also had dysarthria. However, at 6 and 12 month follow-ups the number of physically disabled patients was too small to allow reliable resuts to be obtained. Moreover, many patients were reported to have dysarthria or dysphasia but not to have physical disability.

(3) The emotional category consisted of loss of emotional control (temper, irritability, etc) and loss of emotional stability (mood swings, depression, etc). Emotional changes were rereported very frequently and were often viewed seriously by relatives. Irritability, for example, was reported in $63 \%, 69 \%$ and $71 \%$ of cases at 3, 6 and 12 months respectively. Other difficulties reported in more than half the cases were impatience, tension and anxiety, and depression, at 3 months; and impatience, bad temper, tension and anxiety, depression, and personality change, at both 6 and 12 months. Where bad temper was reported, irritability was also reported but the reverse was not true: it appears that at least some respondents used the former term to denote a greater degree of ill-humour. Even the less frequent items in this area were still reported in a substantial number of cases (for example mood swings were noted in $33 \%$, $38 \%$ and $62 \%$ of cases at consecutive interviews). Whereas physical and language items almost invariably declined in frequency over time, half the items in the emotional group were reported with increasing frequency at consecutive interviews.

(4) Items in the dependence group included difficulties in self-care and the need for supervision. Between $20 \%$ and $25 \%$ "could not be left in charge of the household" even after 1 year but only $17 \%$ needed help with washing and dressing at 3 months and only $9 \%$ by 12 months. Problems in this area usually declined over time.

(5) The subjective category consisted such subjective symptoms as slowness, tiredness, poor concentration, and headaches. These problems were very common, for example $86 \%, 69 \%$ and $67 \%$ reported slowness at 3,6 and 12 months respectively. Around a half reported headaches and over a third reported poor concentration even at 1 year. Nevertheless, difficulties in this area tended to become less frequent over time.

(6) The group of memory items included disorientation, omissions, repetitions and other indications of memory lapses. Overall, memory difficulties were reported in $73 \%$ of cases at 3 months, $59 \%$ at 6 months and $69 \%$ at 12 months.

(7) Finally a group of items designated disturbed behaviour was formed. This consisted of bizarre or puzzling conduct, including violent and inappropriate social behaviour. Threats of violence and inappropriate social behaviour were reported in less than $20 \%$ of cases at each stage. However, the appearance of such phenomena in families where they had previously been absent or markedly less frequent tended to be viewed seriously by relatives. The most frequent changes were the onset of excessive talking $(33 \%, 26 \%$ and $27 \%)$ and childishness $(35 \%, 35 \%$ and $46 \%$ ). Many of the items in the disturbed behaviour category increased or fluctuated in frequency over time with few showing a steady decline.

Changes in sexual adjustment are not considered in detail in this paper because of the difficulty in comparing married or cohabiting patients with those who do not have a partner. However, $15(48 \%)$ of the 31 spouses interviewed reported a change in their partner's sexual behaviour at each follow-up; and where the interviewee was not the spouse there were fewer reports (less than $15 \%$ ) of a change in "interest" in the opposite sex. Changes in sexual adjustment will be examined more fully in later papers.

In order to obtain a clearer overall picture, the number of difficulties reported in each of the seven categories was calculated and the absolute frequency scaled to give a figure "out of 10". A one way analysis of variance was carried out for each follow-up to test the hypothesis that significantly more changes in the patient were reported in some categoires than others. This hypothesis was confirmed $(p<0.01)$ for all three follow-up times and Scheffe tests indicated that a higher number of emotional and subjective items were reported than items in any other category $(\mathrm{p}<0.01)$ at all follow-ups (table 8).

Table 8 Mean number of difficulties (out of 10) reported by relatives

\begin{tabular}{llll}
\hline & 3 months & 6 months & 12 months \\
\hline Physical & 1.8 & 1.6 & 1.5 \\
Subjective & 4.5 & 4.3 & 4.5 \\
Language & 2.4 & $2 \cdot 2$ & $2 \cdot 1$ \\
Emotional & 4.6 & 5.1 & 5.4 \\
Dependence & 1.8 & 1.5 & 1.4 \\
Disturbed behaviour & 1.4 & 1.6 & 1.9 \\
Memory & 2.7 & 2.3 & 2.7 \\
\hline
\end{tabular}


Next, the level of subjective burden (SB) experienced by relatives was considered. On a scale from 1 (no stress) to 7 (severe stress), the mean SB reported was 3.5 at each follow-up, which approximates to "moderate stress." It is possible that SB experienced by relatives is merely a reflection of the severity of injury sustained by the patient. Duration of post-traumatic amnesia (PTA) was used as an index of severity; and cases were divided into three groups according to the degree of stress (subjective burden) experienced by the relative on a seven point scale as shown in table 9. The length of PTA reported in the three groups was compared by means of analysis of variance. At 3 months there was a highly significant $(\mathrm{p}<0 \cdot 001)$ tendency for cases where SB was higher to have longer PTA; at 6 months the differences reached only the 0.05 level of significance; and at 12 months there was a trend which failed to reach statistical significance (table 10).

Table 9 Division of cases into 3 subjective burden $(S B)$ groups according to the degree of $S B$ experienced by the relative

\begin{tabular}{lllll}
\hline & \multicolumn{3}{l}{ Number of cases } \\
\cline { 2 - 5 } \cline { 3 - 4 } & & 3 months & 6 months & 12 months \\
\hline "Low stress" & SB rating of 1-2 & 17 & 15 & 14 \\
"Medium stress" SB rating of 3-4 & 22 & 22 & 20 \\
"High stress" & SB rating of 5-7 & 16 & 18 & 21 \\
\hline
\end{tabular}

Table 10 Comparison by means of one way analysis of variance of the duration of PTA in patients whose relatives experience low, medium, or high stress

\begin{tabular}{llll}
\hline & \multicolumn{4}{l}{ Mean PTA in days } \\
\cline { 2 - 4 } & 3 months & 6 months & 12 months \\
\hline Group 1 (SB=1 or 2) & $13 \cdot 3$ & $14 \cdot 4$ & $17 \cdot 7$ \\
Group 2 (SB=3 or 4) & $24 \cdot 0$ & $33 \cdot 9$ & $31 \cdot 2$ \\
Group 3 (SB=5-7) & $49 \cdot 7$ & $38 \cdot 9$ & $37 \cdot 7$ \\
\hline
\end{tabular}

One way analysis of variance: probability of F0.0002, 0.0359, 1013.

Note The changes in PTA which occur over time are due only to changes in reported SB and consequent changes in the composition of the 3 SB groups. Only one value of PTA was used for each case.

The relationship between objective and subjective burden was examined by means of analysis of variance. Again cases were divided into three groups according to the degree of stress (subjective burden) experienced by the relative as rated on a seven point scale, as shown in table 9. To determine whether relatives who reported higher degrees of stress (SB) also observed more changes in the patient than low stress relatives, the three SB groups were compared on the seven problem areas by means of one way analysis of variance.
A significance level of 0.01 was used to reduce the risk of type 1 error.

For almost all problem areas and follow-ups the trend is that the higher the perceived stress, the more changes in the patient are reported although this is not always statistically significant (fig 2). No significant differences were found between the three SB groups on the number of changes in the physical and language categories. Significant differences were found between SB groups on the number of subjective. emotional and disturbed behaviour changes at all follow-ups. The differences between SB groups on the number of
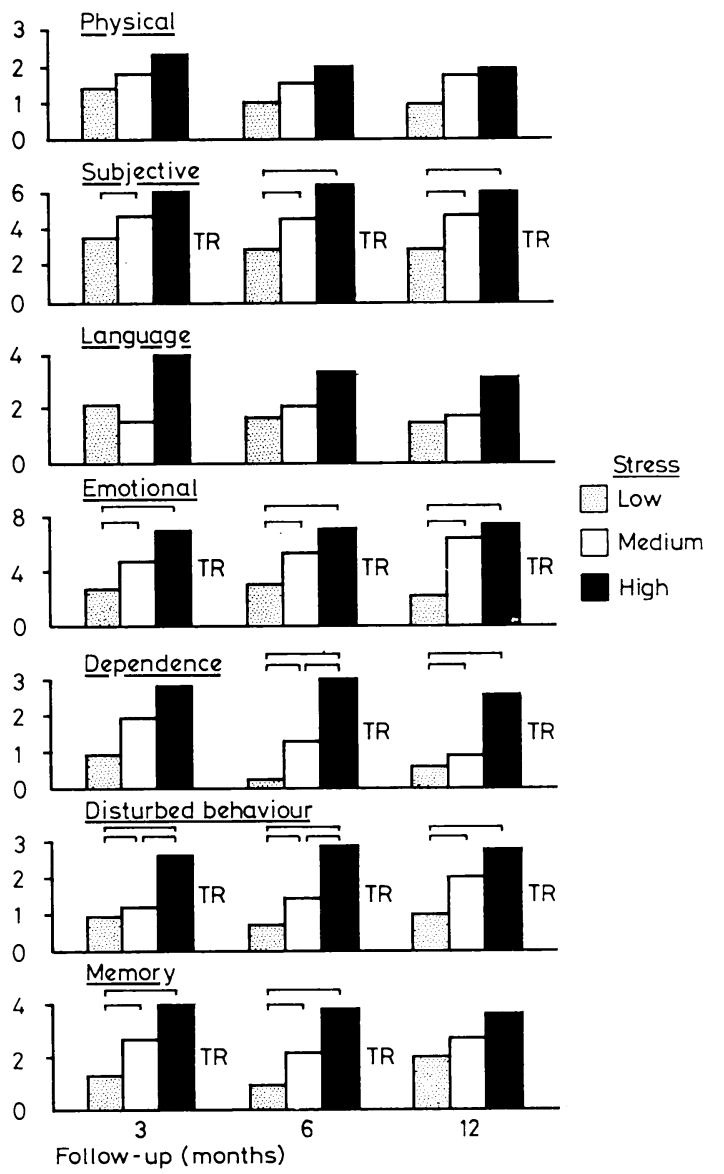

Fig 2 Mean number of changes in the patient reported by relatives under low, medium and high stress. Results are given for the seven problem areas at each follow-up time. "TR" (trend) denotes a significant $(p<0.01)$ one way analysis of variance across groups; and pairs of groups which are bracketed together differ significantly $(p<0.01)$ from each other. 
memory changes were significant at 3 and 6 months; and the differences between SB groups on the number of dependence changes were significant at 6 and 12 months.

Where the one way analysis of variance was significant, all three groups did not always differ significantly. For example, "low stress" relatives reported fewer emotional changes in the patient than "medium" or "high" stress relatives, but these last two did not differ significantly at all three follow-ups.

Finally, the role of compensation claims was considered as a possible factor in elevating the number of difficulties reported. From the sample of 55 cases, two subsamples of 21 were chosen. These were cases who had consistently, at each follow-up, expressed the view that they had grounds to pursue a claim, or that they had no such grounds. Cases where there was some doubt and the two cases where the claim was settled were excluded. The two groups were compared in three ways. Firstly, a series of $t$-tests were carried out comparing the number of reported changes in the patients in each of the seven problem areas already outlined. None of the 21 comparisons reached the $5 \%$ level of significance. Secondly, there was a tendency for cases claiming compensation to be more severely injured in terms of PTA (claim group: mean PTA $=39$ days; no-claim group: mean PTA $=18$ days) although because of large variances this failed to reach the $5 \%$ level of significance on the $t$-test. Thirdly, a series of analyses of variance were caried out, using PTA as covariate. Again there were no significant differences at the $5 \%$ level between claim and no-claim groups.

\section{Discussion}

Our results indicate that for a group of adults with severe blunt head injury the problems most frequently reported by relatives are emotional changes, poor memory and subjective symptoms such as slowness and tiredness. Physical difficulties are less frequent and all but five out of 55 cases were independently mobile without the need of a stick, crutch or wheelchair by 6 months after injury. There was a tendency for certain types of problem, particularly emotional difficulties to be reported more frequently at later follow-ups: for example, bad temper was reported in $48 \%$ of cases at 3 months and $67 \%$ at 12 months; and mood swings were reported in $33 \%$ at 3 months and $62 \%$ at 12 months. It may be that these changes are a reaction to disability, that is, they stem from frustration at not being able to resume normal activities and at not being free from symptoms; or it may be that they are a primary result of the injury and although present from the time of injury are not observed or admitted by the relatives for some time, as suggested by Romano ${ }^{12}$ and Lezak. ${ }^{13}$ To try to resolve this issue, amongst others, we are at present collecting data on a group of paraplegic patients: these are patients whose lives have been threatened and who have been disabled by trauma but who have escaped brain injury. When these data are available we hope to return to the issue.

Compensation did not play a significant role in elevating the number of changes reported in the patients. There was a slight (but statistically insignificant) tendency for those claiming compensation to have had more severe injuries. However, whether or not this is allowed for (by means of covariance analysis) there are no statistically significant differences in the number of changes reported in cases where compensation is claimed and those where it is not claimed. Indeed, the differences in the number of changes reported in the two groups is very small and occurs in both directions.

The mean level of stress experienced by relatives was the same at 3,6 and 12 months, postinjury which is consistent with the finding of Oddy et $a l,{ }^{11}$ that stress levelled off sometime between 1 and 6 months. The relationship between the severity of the patient's injury (as assessed by PTA), and the degree of stress experienced by the relative weakened over time: stress in relatives is not a simple reflection of severity of injury. However, there is an association between stress experienced by the relatives and certain types of reported problems in the patient. The specific relationships have already been outlined, but in general, mental and behavioural changes in the patient are associated with significantly increased stress in the relative whereas physical and speech/ language difficulties are not. It should not be assumed that there is a direct causal relationship between reported changes in the patient (objective burden) and the stress which the relative suffers and attributes to these changes (subjective burden). Both types of burden are assessed on the basis of a relative's report and there may well be an interplay between stress in the relative and the relative's observation of the patient whereby the observations are coloured by the degree of stress experienced. For this reason the conclusion of Oddy et $a l^{11}$ that personality change is a "source" of stress for relatives should be treated 
with caution since they used a method similar to ours in that reports of patients' problems and relatives' stress were both obtained from relatives. However, to the extent that we have found a link between reported mental and behavioural changes in the patient and stress in the relative our findings are in broad agreement with previous studies $^{71}$ as well as our own previous work. ${ }^{24}$ This has important implications for rehabilitation. The fact that an association exists between reports of mental and behavioural changes in the patient and stress in the relative indicates that in those families most in need of help, rehabilitationists will find that mental and behavioural problems are the primary concern.

In conclusion, we have provided a description of the problems of adults with severe blunt head injury in the 12 months after injury as seen by close relatives. An account has also been given of those types of problem most associated with stress in the relative. Future analysis will describe the resumption of work and other activities in the same group of patients and the link between the persistence of the problems discussed in this paper and the resumption of normal activities.

The investigation was supported by the Medical Research Council, Grant No G/975/928.

We are grateful to the Consultant Neurosurgeons at The Institute of Neurological Sciences, Southern General Hospital, Glasgow, who allowed their patients to be studied.

\section{References}

1 London PS. Some observations on the course of events after severe injury of the head. $A n n R$ Coll Surg Engl 1967; 41:460-9.

2 Bond MR. Assessment of the psychosocial outcome after severe head injury. In: Outcome of severe damage to the CNS. Ciba Foundation Symposium, 1975; 34:141-53.

3 Bond MR, Brooks DN. Understanding the process of recovery as a basis for the investigation of rehabilitation for the brain-injured. Scand J Rehabil Med 1976; 8:127-33.

4 Brooks DN, Aughton ME. Psychological consequences of blunt head injury. Int Rehabil Med 1979; 1:160-5.

5 Schacter DL, Crovitz HF. Memory function after closed head injury: A review of the quantitative research. Cortex 1977; 13:150-76.
6 Fahy TJ, Irving MH, Millac P. Severe head injuries: a six year follow-up. Lancet 1967; 475-9.

7 Thomsen IV. The patient with severe head injury and his family. Scand J Rehabil Med 1974; 6:180-3.

8 Rosenbaum $M$, Najenson $T$. Changes in life patterns and symptoms of low mood as reported by wives of severely brain-injured soldiers. J Consult Clin Psychol 1976; 44:881-8.

9 Lezak MD. Living with the characterologically altered brain injured patient. J Clin Psychiatry 1978; 39:592-8.

10 Panting A, Merry P. The long term rehabilitation of severe head injuries with particular reference to the need for social and medical support for the patient's family. Rehabilitation (Stuttg) 1972; 38:33-7.

11 Oddy M, Humphrey M, Uttley D. Stresses upon the relatives of head-injured patients. $B r J$ Psychiatry 1978; 133:507-13.

12 Romano MD. Family response to traumatic head injury. Scand J Rehabil Med 1974; 6:1-4.

13 Lezak MD, Cosgrove JN, O'Brien K, Wooster N. Relationships between personality disorders, social disturbances and physical disability following traumatic brain injury. Paper presented at the Eighth Annual Meeting of the International Neuropsychological Society, San Francisco, 1980.

14 Steadman JH, Graham JG. Head injuries: an analysis and follow-up study. Proc $R$ Soc Med 1970; 63:23-8.

15 Oddy M, Humphrey M, Uttley D. Subjective impairment and social recovery after closed head injury. J Neurol, Neurosurg Psychiatry 1978; 41:611-6.

16 Jennett B, Bond M. Assessment of outcome after severe brain damage: a practical scale. Lancet $1975 ; 480-4$.

17 Brooks DN. Psychological deficits after severe blunt head injury: their significance and rehabilitation. In: Oborne DJ, Gruneberg MM, Eiser JR, eds. Research in Psychology and Medicine, Volume 2. London: Academic Press 1979; 469-76.

18 Field JH. Epidemiology of head injuries in England and Wales. London: Her Majesty's Stationery Office, 1976.

19 Grad J, Sainsbury P. Evaluating a community care service. In: Freeman H, Farndale WL, eds. Trends in the Mental Health Services. London: Pergamon, 1963: 303-17.

20 Hoenig J, Hamilton MW. The Desegregation of the Mentally Ill. London: Routledge \& Kegan Paul, 1969. 